\title{
Erratum to: Prokaryotic expression, purification and functional characterization of recombinant human RIP2
}

\author{
Xin Cai $\cdot$ Min Wang $\cdot$ Haibo Kong $\cdot$ Jing Liu $\cdot$ Ye Liu \\ Wengrong Xia $\cdot$ Minji Zou $\cdot$ Jiaxi Wang $\cdot$ Hang Su $\cdot$ \\ Donggang Xu
}

Published online: 28 November 2012

(C) Springer Science+Business Media Dordrecht 2012

\section{Erratum to: Mol Biol Rep}

DOI 10.1007/s11033-012-1995-z

An acknowledgement has been added to the existing publication:

Acknowledgments This research was supported by Grants from the Major State Basic Research Development Program of China (973 Program) (No. 2011CB503803) and National Natural Science Foundation of China (No. 30873030, No. 81071928, No. 81001175 and No. 81071945).

The online version of the original article can be found under doi:10.1007/s11033-012-1995-z.

X. Cai $\cdot$ M. Wang $\cdot$ Y. Liu $\cdot$ W. Xia $\cdot$ M. Zou $\cdot$ J. Wang D. Xu (凶)

Laboratory of Genome Engineering, Beijing Institute of Basic

Medical Sciences, Beijing 100850, China

e-mail: xudg@nic.bmi.ac.cn

H. Kong

Department of Neurosurgery, The Second Hospital of Hebei

Medical University, Shijiazhuang 050000, China

J. Liu · H. Su ( $₫)$

Affiliated Hospital of Military Medical Science Academy,

Beijing 100071, China

e-mail: suhang_lr@sohu.com 